\title{
Índices Alternativos del WISC-IV para la Evaluación del Razonamiento Fluido
}

\author{
WISC-IV Alternative Indexes for the Assessment of Fluid Reasoning Abilities
}

\author{
Agustina Labin ${ }^{1}$, María Elena Brenlla ${ }^{2}$ y Alejandra Taborda ${ }^{3}$
}

\begin{abstract}
Resumen
A partir de las investigaciones sobre las capacidades cognitivas evaluadas por la Escala de inteligencia de Wechsler para niños y adolescentes (WISC-IV), y las aptitudes específicas que propone la teoría de inteligencia de Cattell-Horn-Carroll (CHC), en este artículo se presentan dos índices alternativos del WISCIV que permiten una comparación adicional y favorece la construcción de hipótesis potencialmente significativas sobre las habilidades cognitivas fluidas del examinado. Se trabajó con la muestra de tipificación de Buenos Aires, Argentina $(\mathrm{n}=1437)$ del WISC-IV. Se crearon los índices de razonamiento fluido verbal (Gf-v) y no verbal (Gf-nv), un registro para la implementación de los mismos y las tablas de conversión correspondientes. Por último, se concluye que se trata de estimaciones útiles que permiten ampliar la lectura diagnóstica que cada perfil delinea, favorece el análisis exhaustivo del índice de razonamiento perceptivo del WISC-IV y específicamente, enriquecen el estudio de la capacidad fluida.
\end{abstract}

Palabras clave: WISC-IV, índice razonamiento fluido no verbal, índice razonamiento fluido verbal, CHC

\begin{abstract}
In this article, we present two optional WISC-IV indexes that both allow for an additional comparison, and favor the formulation of potentially significant hypotheses on the fluid cognitive abilities of the candidate. Such indexes derived from research on the cognitive capacities assessed by the Wechsler Intelligence Scale for children (WISC-IV) and on the specific aptitudes proposed by the theory of Cattell-Horn-Carroll (CHC) on intelligence. A WISC-IV classification sample from Buenos Aires, Argentina $(\mathrm{n}=1437)$ was selected. Verbal (Gf-v) and non-verbal (Gf-nv) fluid reasoning indexes, a register for the implementation of those indexes and the corresponding conversion tables were created. Finally, we conclude that these are useful estimations that allow for the diagnostic reading assessment of each profile, favor the exhaustive analysis of the WISC-IV perceptual reasoning index measures, and above all, enrich the study of fluid capacity.
\end{abstract}

Keywords: WISC-IV, non-verbal fluid reasoning index, verbal fluid reasoning index, CHC

\footnotetext{
${ }^{1}$ Lic. en Psicología. Facultad de Psicología, Universidad Nacional de San Luis, Argentina. CCT- CONICET San Luis. Jefe de trabajos prácticos - Psicología Educacional. Av. Ejercito de los Andes 950 (5700) San Luis, Argentina. Tel.: 2664641432. Correo: alabin@unsl.edu.ar

${ }^{2}$ Dra. en Psicología. Centro de Investigaciones en Psicología y Psicopedagogía (CIPP), Argentina. Facultad de Psicología y Psicopedagogía, Pontificia Universidad Católica, Argentina. Correo: bren@uca.edu.ar

${ }^{3}$ Dra. en Psicología. Facultad de Psicología, Universidad Nacional de San Luis, Argentina. Profesor Titular - Psicología Educacional. Av. Ejercito de los Andes 950 (5700) San Luis, Argentina. Correo: ataborda@unsl.edu.ar
} 


\section{Introducción}

En las últimas décadas el abanico y refinamiento de los aportes psicométricos permiten sistematizar evidencias empíricas que sustentan nuevos desarrollos teóricos y en circularidad impactan en el terreno de la investigación, prevención y asistencia de la salud mental. De este modo, la complejidad implicada en el diagnóstico de la capacidad intelectual de niños y adolescentes, es una de las temáticas que a lo largo de la historia ha dado origen a una multiplicidad de investigaciones que desembocan en la construcción de modelos explicativos e instrumentos de evaluación. A pesar de ello la problemática se encuentra lejos de estar zanjada, las vicisitudes de la singularidad humana, contextual e histórica, ponen en evidencia los alcances y limitaciones de las pruebas psicométricas. Una de las mayores dificultades se presenta a la hora de interpretar perfiles que delinean discrepancias significativas. Más aún si se tiene en cuenta que, en la vida de una persona el diagnóstico nunca resulta neutro es terapéutico o iatrogénico y es tan iatrogénico pasar por alto habilidades genuinas como aquellos diagnósticos que muestran puntos ciegos en la comprensión de las dificultades cognitivas (Coronel, Lacunza, \& Contini, 2006; Taborda, 2013). Los estudios sobre plasticidad neuronal abren nuevos caminos y con ello, demanda de mayores profundizaciones en el estudio de fortalezas y limitaciones (Carasatorrea, Ramírez-Amaya, \& Díaz Cintra, 2016; Muñoz Céspedes, Tirapu Ustárroz, Ríos Lago, \& Paúl Lapedriza, 2008).

En este escenario, el análisis e interpretación de las discrepancias de las puntuaciones obtenidas en las pruebas psicométricas toman una peculiar relevancia. Para alcanzar tales objetivos, resulta central trabajar con tests psicológicos minuciosamente estudiados en su estructura interna; coherencia y puesta al día con los avances en las operacionalizaciones del polifacético constructo capacidad intelectual; especificidades evolutivas; validez y fiabilidad tanto de las normas de administración como de la evaluación psicométrica e interpretativa. En otras palabras, verificar si reúnen las condiciones éticas, teóricas y metodológicas necesarias para su implementación.
La cuarta generación del test de Wechsler (WISC-IV) para la evaluación de la capacidad intelectual de niños y adolescentes, instituye una nueva propuesta que condensa reconceptualizaciones sobre las funciones cognitivas elaboradas en base a los avances en neurociencias y el modelo de inteligencia fluida y cristalizada (Flanagan \& Kaufman, 2009; Taborda, Barbenza, \& Brenlla, 2011). Además, incorpora actualizaciones de los estímulos, modificaciones en las pautas de administración, progresos en la metodología psicométrica y considera las nuevas normas que se desprenden de la legislación señaladas en el Acta sobre Educación de Personas con Discapacidad (IDEA). A su vez, los cambios se reflejan en el abandono de la tradicional clasificación de CI verbal y de ejecución, en procura de evaluar los dominios cognitivos específicos implicados en los índices comprensión verbal (ICV), razonamiento perceptivo (IRP), memoria operativa (IMO) y velocidad de procesamiento (IVP), que a su vez se reúnen para la evaluación del cociente intelectual total (CIT).

La bibliografía actual recomienda integrar las ampliaciones interpretativas del WISC-IV al proponer un enfoque ipsativo de las áreas fuertes y débiles del perfil individual con el normativo focalizado en la comparación interindividual con referencias a normas que incluyen más acabadamente el modelo jerárquico de la teoría CHC, sustentado en la combinación de los aportes de Cattell, Horn y Carroll (Flanagan \& Kaufman, 2009; Flanagan, Ortiz, Alfonso, \& Dynda, 2014; McGrew, 2009).

Cattell (1963) introdujo la noción de inteligencia conformada por dos factores: a) la inteligencia fluida y b) la inteligencia cristalizada. La primera, es independiente de las experiencias culturales de los sujetos e implica capacidades de razonamiento abstracto y resolución de problemas más allá de los conocimientos adquiridos (Ferrer, O'Hare, \& Bunge, 2009; Keith \& Reynolds, 2012). Entre tanto, la inteligencia cristalizada puede ser pensada como el conocimiento cultural incorporado a través de un proceso de aculturación y experiencias socioeducativas previas para su posterior aplicabilidad. Lo cristalizado remite tanto el conocimiento declarativo que requiere de la memoria a largo 
plazo como el procedimental que conlleva procesos de razonamiento con operaciones aprendidas previamente para transformar el conocimiento. Por lo tanto, funciona como un almacén de conocimientos y simultáneamente como un conjunto de capacidades de procesamiento (Flanagan \& Kaufman, 2009; McGew, 2009). Años más tarde Horn, discípulo de Cattell, amplió la teoría y señaló la relevancia de los factores percepción visual, memoria de corto plazo y almacenamiento y recuperación a largo plazo, entre otros (Horn \& Noll, 1997; Horn, 1991). Por su parte, Carroll (1993), comparó los resultados de distintos análisis factoriales de las medidas de habilidades cognitivas existentes y concluyó que la evidencia de un factor general de inteligencia era abrumadora. Asimismo, coincidió con Cattell y Horn en aceptar que la inteligencia tiene una estructura jerárquica, que se compone de habilidades específicas que aparecen en dominios de habilidades cognitivas de nivel superior y le otorgó especial relevancia a los procesos de razonamiento fluido (Carroll, 1993; Keith, 1990).

En consecuencia, desde esta perspectiva las habilidades intelectuales se organizan jerárquicamente en tres estratos: a) estrato general, b) estrato amplio y c) estrato próximo. El primero designa a una habilidad intelectual que, en términos psicométricos, se corresponde con el factor $\mathrm{g}$; el segundo refiere a habilidades amplias $\mathrm{y}$, el tercero, contiene cerca de 60 componentes que denotan habilidades limitadas y se subsumen en el anterior. La mayoría de las investigaciones se ha centrado en el segundo estrato que refiere a las habilidades de mayor amplitud que incluye la inteligencia cristalizada, el razonamiento fluido, los procesamientos visual y auditivo, la memoria de corto plazo y largo plazo, la velocidad de procesamiento y el conocimiento cuantitativo (Flanagan \& Kaufman, 2009).

La investigación realizada sobre la adaptación y estandarización argentina del WISC-IV, sustentada en estudios de análisis factorial confirmatorio señala que el modelo alternativo basado en la teoría $\mathrm{CHC}$ de un factor $\mathrm{g}$ y cinco grupos resulta un enfoque sustantivo para realizar la interpretación del WISC-IV en nuestro medio. Las habilidades del estrato amplio $\mathrm{CHC}$ puestas en juego en la resolución de las situaciones problemáticas que presentan los estímulos del
WISC-IV incluyen la inteligencia cristalizada, el procesamiento visual, el razonamiento fluido, la memoria de corto plazo y la velocidad de procesamiento. El estudio factorial realizado en nuestra población difiere del estudio norteamericano, al no considerar el subtest aritmética (Ar) como una medida de razonamiento fluido. Aquí se encontró que matrices (M) y conceptos (Co) cargan sobre razonamiento fluido, mientras que Ar presenta cargas compartidas con la inteligencia cristalizada y con la memoria a corto plazo (Brenlla, 2013; Keith, Fine, Taub, Reynolds, \& Kranzler, 2006).

Desde esta perspectiva y con el propósito de aportar nuevas herramientas psicométricas focalizadas en los procesos de pensamiento fluido, el objetivo de este artículo es diseñar dos puntuaciones índices del WISC-IV que permitan ampliar el análisis e interpretación de discrepancias: el índice de razonamiento fluido no verbal (Gf-nv) ${ }^{1}$ y el índice de razonamiento fluido verbal (Gf-v), elaborados sobre los datos de la muestra de tipificación de Buenos Aires (Argentina) (Taborda, Barbenza, \& Brenlla, 2011).

La literatura vigente sobre la Escala de Wechsler, define a las habilidades fluidas (Gf), como la instrumentación de las operaciones mentales para resolver problemas novedosos que no pueden ser solucionados de manera automática. Estas operaciones pueden incluir formación y reconocimiento de conceptos, percepción de las relaciones entre patrones, elaboración de conclusiones, comprensión de implicaciones, solución de problemas, y restauración o reorganización de información. Los procesos de razonamiento inductivo y deductivo son los indicadores de las habilidades específicas fluidas evaluadas a través de subtests que requieren identificar patrones de relaciones abstractas entre estímulos verbales y no verbales (Flanagan \& Kaufman, 2009; Weiss, Saklofske, Holdnack, \& Prifitera, 2015).

Para ponderar el conjunto Gf-nv se recurre a los subtests matrices (M) y conceptos (Co) que implican el uso de estímulos visuales y no requieren lenguaje expresivo. En tanto, el índice Gf-v comprende semejanzas (S) y adivinanzas

\footnotetext{
${ }^{1}$ Los códigos de dos letras (ej. Gf-nv) son de Carroll (1993)
} 
(Ad) que implican la amplitud y profundidad del conocimiento acumulado así como su efectiva utilización.

\section{Método}

\section{Participantes}

Para la construcción de los índices se utilizaron los datos de la muestra de tipificación de Buenos Aires, Argentina compuesta por 1437 niños y adolescentes. En el muestreo se consideraron tres variables para la estratificación, el sexo, la edad y la zona. Se consultaron los datos del Censo (Instituto Nacional de Estadísticas y Censos [INDEC], 2001) acerca de la población escolar en cada región y, se calculó para cada uno de los grupos de edad la cantidad de casos según sexo, edad y región que debían recolectarse. La misma refleja la población infanto-juvenil de entre 6 y 16 años residentes de la Ciudad Autónoma de Buenos Aires y el Gran Buenos Aires (Argentina). La muestra estuvo formada de un número semejante de varones y mujeres en cada grupo de edad, divididos en 11 grupos: 6:0-6:11, 7:0-7:11, 8:0-8:11, 9:0-9:11, 10:0-10:11, 11:0-11:11, 12:012:11, 13:0-13:11, 14:0- 14:11, 15:0-15:11, 16:016:11. Cada grupo se conformó de 120 a 135 participantes. De la muestra total, el $50.4 \%$ fueron niñas y el $49.6 \%$, niños. En cuanto a la zona de residencia, el $34.9 \%$ fue de partidos de la zona sur, el $34.7 \%$ de la zona norte y el 30.2 de la zona oeste del Gran Buenos Aires.

Se establecieron como criterios de exclusión a los siguientes: a) haber sido evaluado mediante cualquier instrumento de medición de inteligencia en los últimos 6 meses; b) dificultades visuales y auditivas sin corregir o limitantes; c) falta de fluidez verbal; c) fallos severos en la expresión verbal o de comunicación; d) problemas motores en extremidades superiores; e) estar en tratamiento psiquiátrico, psicológico o psicofarmacológico y, f) diagnóstico previo de alguna enfermedad que pueda disminuir el desempeño en el test.

\section{Instrumento}

Se administró la Escala de inteligencia para niños de Wechsler - versión argentina (WISC-IV), una prueba de administración individual para niños de 6 años y 0 meses a 16 años y 11 meses.
La misma se conforma por 10 subtests principales y 5 subtests complementarios. Las puntuaciones principales permiten calcular el CIT y los cuatro índices compuestos. El ICV se calcula a partir de la suma de las puntuaciones de los subtests: semejanzas (S), vocabulario (V) y comprensión (C). El IRP se obtiene a partir de la suma de las puntuaciones de construcción con cubos (CC), conceptos (Co) y matrices (M). Por su parte, el IMO se calcula a partir de la suma de las puntuaciones de los subtests; retención de dígitos (RD) y letras y números (LyN). El IVP se alcanza a partir de la suma de las puntuaciones de claves (Cl) y búsqueda de símbolos (BS).

Se trata de una prueba que presenta evidencias satisfactorias de validez y fiabilidad tanto en EE.UU (Wechsler, 2003), en España (Wechsler, 2005) como en la Argentina (Taborda, Barbenza, \& Brenlla, 2011). Las evidencias de consistencia interna, a partir del método de división por mitades, muestran coeficientes entre .79 y .90 y los coeficientes de estabilidad test-retest, entre .77 y .86. Presenta adecuada discriminación entre grupos clínicos y evidencias satisfactorias de validez interna. Se observó que de los cinco modelos propuestos para el análisis de las puntuaciones del test, el más consistente resultó el de cuatro factores (Brenlla, 2013).

\section{Procedimiento}

La administración del test se realizó individualmente en dos encuentros de 45 minutos aproximadamente cada uno. En este artículo, únicamente se analizan las puntuaciones obtenidas en los subtests principales $\mathrm{M}, \mathrm{Co}, \mathrm{S}$ y el subtest opcional Ad para la construcción de los dos índices.

Para la construcción del Gf-nv se trabajó con los casos en que la diferencia de $\mathrm{M}$ y Co fuera menor a 5 puntos $(1.5 \mathrm{DE}=3+1.5=4.5 \approx 5)$. Se eliminaron 213 casos y se conservó el resto $(\mathrm{n}=1224)$. Luego se sumaron las puntuaciones escalares de los dos subtests principales y su resultado se convirtió a puntuaciones z. Estas fueron transformadas en puntuaciones compuestas con una $\mathrm{M}=100$ y $\mathrm{DE}=15(\mathrm{z} * 15+100)$. Para la construcción del Gf-v se analizaron los casos en que la diferencia de $\mathrm{S}$ y Ad es menor a 5 puntos, con lo cual se eliminaron 138 casos y se conservó el resto $(n=1299)$. Al igual que en el índice 
anterior, se sumaron las puntuaciones escalares del subtest principal $\mathrm{S}$ y el subtest optativo Ad. Luego, se convirtieron a puntuaciones $\mathrm{z}$, las cuales fueron transformadas en puntuaciones compuestas con una $\mathrm{M}=100$ y $\mathrm{DE}=15$ ( $\mathrm{z} * 15+100)$.

Para obtener evidencias de validez de estructura interna de ambos índices, se realizó un análisis factorial confirmatorio, utilizando Máxima Verosimilitud como método de estimación. Se consideraron múltiples indicadores para evaluar el ajuste del modelo: el estadístico chi-cuadrado, el índice de ajuste comparativo (CFI), el índice de bondad de ajuste (GFI), y el error cuadrático medio de aproximación (RMSEA). Para evaluar los resultados obtenidos por cada índice se utilizaron las recomendaciones y los puntos de cortes establecidos por $\mathrm{Hu}$ y Bentler (1995) y Hair, Black, Babin, Anderson y Tatham (2006). Posteriormente se calcularon los coeficientes alpha de Cronbach para estimar la consistencia interna de los índices siguiendo los criterios de George y Mallery (2003). Se presentan estadísticos descriptivos (media y desvío estándar) para ambos índices y correlaciones entre los mismos, así como con el CIT utilizando el coeficiente de correlación producto-momento de Pearson.

\section{Resultados}

El análisis factorial confirmatorio da como resultado un modelo con un coeficiente chicuadrado no significativo (.013; sig.=.909), y con índices de ajuste considerados excelentes $(\mathrm{CFI}=1$ $\mathrm{GFI}=1$, RMSEA=.00) evidenciando que la estructura interna de los índices propuestos posee un ajuste perfecto. Los coeficientes de regresión estandarizados fueron significativos y variaron entre .82 y .62 (Figura 1).

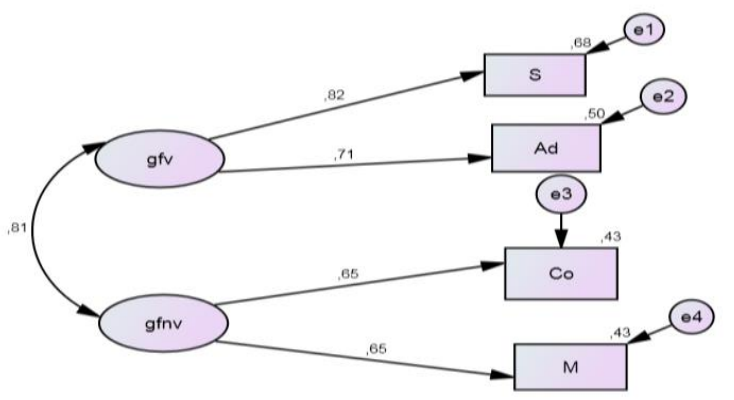

Figura 1. Modelo final con sus pesos de regresión estandarizados
Por otro lado, los estudios de fiabilidad indicaron una adecuada consistencia interna para ambos índices siendo $\alpha=.87$ para Gf-nv y $\alpha=.83$ para Gf-v.

Una vez obtenida evidencia de validez y fiabilidad, se realizaron estudios descriptivos de los componentes de los índices (Tabla 1) y se calcularon correlaciones entre los mismos. Como puede observarse en la Tabla 2, las correlaciones fueron significativas, altas y positivas entre todos los componentes, siendo la relación más fuerte entre $\mathrm{S}$ y $\mathrm{Ad}(\mathrm{r}=.89)$ denotando que el factor fluido verbal es más robusto que el fluido no verbal compuesto por Co y $\mathrm{M}(\mathrm{r}=.83)$.

Tabla 1. Estadísticos descriptivos de los componentes de Gf-nv y Gf-v

\begin{tabular}{lcccc}
\hline & M & DE & Min. & Máx. \\
\hline Semejanzas & 20.48 & 9.29 & 0 & 41 \\
Adivinanzas & 14.72 & 4.52 & 0 & 24 \\
Conceptos & 16.78 & 4.55 & 0 & 27 \\
Matrices & 19.34 & 6.67 & 3 & 33 \\
\hline
\end{tabular}

M=Media: DE=Desvió estándar; Min.=Valor mínimo obtenido; Max-=Valor máximo obtenido

Tabla 2. Correlación entre los subtests que conforman los índices Gf-nv y Gf-v

\begin{tabular}{lcccc}
\hline & Semejanzas & Conceptos & Matrices & Adivinanzas \\
\hline Semejanzas & 1 & & & \\
Conceptos & $.74 * *$ & 1 & & \\
Matrices & $.78 * *$ & $.83 * *$ & 1 & \\
Adivinanzas & $.89 * *$ & $.72 * *$ & $.77 * *$ & 1 \\
\hline
\end{tabular}

Posteriormente, se analizó la correlación entre ambos índices con el CIT que arroja el WISC-IV. Se obtuvieron correlaciones altas, positivas y significativas entre el CIT y el Gf-nv ( $\mathrm{r}=.77$; $p \leq .001)$; y entre el CIT y el Gf-v ( $\mathrm{r}=.76 ; p \leq .001)$.

Por último, se conformaron los baremos de interpretación. La tabla 3 permite observar la conversión del índice y sus percentiles a partir de las sumas de $\mathrm{M}$ y $\mathrm{Co}$, mientras que la tabla 4 presenta las puntuaciones índices Gf-v a partir de la suma de $\mathrm{S}$ y Ad. Los índices se alcanzaron mediante la técnica estadística de ajuste lineal (Tellegen \& Briggs, 1967).

Asimismo, se establecieron recomendaciones para la implementación de los índices alternativos de razonamiento fluido del WISC-IV y se diseñó un protocolo de registro interpretativo para los 
Tabla 3. Tabla de conversión del Gf-nv basada en las sumas de las puntuaciones escalares de los subtests principales M y Co del WISC-IV: normas argentinas

\begin{tabular}{lcclcc}
\hline $\begin{array}{l}\text { Suma de las } \\
\begin{array}{l}\text { Puntuaciones } \\
\text { Escalares }\end{array}\end{array}$ & Gf-nv & $\mathrm{P}$ & $\begin{array}{l}\text { Suma de las } \\
\text { puntuaciones } \\
\text { escalares }\end{array}$ & Gf-nv & $\mathrm{P}$ \\
\hline 6 & 58 & $<1$ & 21 & 102 & $51-59$ \\
7 & 61 & $<1$ & 22 & 105 & $60-67$ \\
8 & 64 & 1 & 23 & 108 & $68-73$ \\
9 & 67 & 2 & 24 & 111 & $74-79$ \\
10 & 70 & 2 & 25 & 114 & $80-83$ \\
11 & 72 & $3-4$ & 26 & 116 & $84-88$ \\
12 & 75 & 5 & 27 & 119 & $89-90$ \\
13 & 78 & $6-8$ & 28 & 122 & $91-93$ \\
14 & 81 & $9-12$ & 29 & 125 & $94-95$ \\
15 & 84 & $13-17$ & 30 & 128 & $96-97$ \\
16 & 87 & $18-22$ & 31 & 131 & 98 \\
17 & 90 & $23-27$ & 32 & 134 & 99 \\
18 & 93 & $28-35$ & 34 & 140 & $>99$ \\
19 & 96 & $36-42$ & 35 & 143 & $>99$ \\
20 & 99 & $43-50$ & 36 & 146 & $>99$ \\
\hline
\end{tabular}

Tabla 4. Tabla de conversión del Gf-v basada en las sumas de las puntuaciones escalares del subtest principal S y del subtest optativo Ad del WISC-IV: normas argentinas

\begin{tabular}{lcclcc}
\hline $\begin{array}{l}\text { Suma de las } \\
\text { puntuaciones } \\
\text { escalares }\end{array}$ & Gf-v & $\mathrm{P}$ & $\begin{array}{l}\text { Suma de las } \\
\text { puntuaciones } \\
\text { escalares }\end{array}$ & Gf-v & $\mathrm{P}$ \\
\hline 3 & 52 & $1-2$ & 20 & 99 & $44-52$ \\
4 & 54 & $1-2$ & 21 & 102 & $53-59$ \\
5 & 57 & $1-2$ & 22 & 105 & $60-67$ \\
6 & 60 & $1-2$ & 23 & 108 & $68-73$ \\
7 & 63 & $1-2$ & 24 & 111 & $74-79$ \\
8 & 66 & $1-2$ & 25 & 113 & $80-84$ \\
9 & 68 & $1-2$ & 26 & 116 & $85-88$ \\
10 & 71 & 3 & 27 & 119 & $89-90$ \\
11 & 74 & $4-5$ & 28 & 122 & $91-93$ \\
12 & 77 & $6-7$ & 29 & 125 & $94-95$ \\
13 & 80 & $8-10$ & 30 & 127 & 96 \\
14 & 82 & $11-14$ & 31 & 130 & 97 \\
15 & 85 & $15-19$ & 32 & 133 & 98 \\
16 & 88 & $20-24$ & 33 & 136 & 99 \\
17 & 91 & $25-30$ & 34 & 139 & $>99$ \\
18 & 94 & $31-36$ & 35 & 141 & $>99$ \\
19 & 97 & $37-43$ & & & \\
\hline
\end{tabular}

mismos (ver Material Complementario). Con el sustento de la muestra alcanzada en Argentina, se propone 23 puntos como diferencia significativa entre Gf-nv y Gf-v (independientemente de su dirección) que se comparece con menos del 10\% de la población.

\section{Discusión}

Las últimas investigaciones sobre habilidades cognitivas específicas desde la teoría CHC, ofrecen un modelo para pensar en nuevas lecturas 
sobre las capacidades del examinado. En este marco, la creación de dos índices de razonamiento fluido permite realizar comparaciones interpretativas opcionales que favorecen la ampliación de la información obtenida en el análisis del perfil que arroja el WISC-IV (Brenlla, Taborda, \& Labin, 2013; Flanagan \& Kaufman, 2009; Frank de Verthelyi, 1999; McGrew, 2009).

Esta ampliación permite comparar dos pares de subtests o conjuntos clínicos. Para ello, el examinador puede planificar previamente realizar estas comparaciones clínicas o a posteriori de la evaluación, no obstante para poder efectuar este análisis es necesario aplicar el subtest optativo Ad. Se pueden realizar comparaciones clínicas cuando los dos conjuntos a contrastar encarnen aptitudes unitarias, representadas por un conjunto cohesionado de puntuaciones escalares. Cuando la variabilidad de los puntajes escalares de los subtests que componen los índices Gf-nv y Gf-v es menor que $1.5 \mathrm{DE}$ (5 puntos), es pertinente considerar que el índice representa una estimación unitaria, fiable y válida, y de este modo permite su instrumentación e interpretación. Por lo tanto, antes de analizar el desempeño del examinado en estas puntuaciones índices, es necesario determinar si el mismo es interpretable. Si un índice resulta no interpretable, la información obtenida a partir del desempeño en la prueba tiene que basarse en los resultados individuales obtenidos en cada uno de los subtests.

Específicamente, el índice Gf-nv se compone de $M$ que evalúa el razonamiento deductivo a partir de una secuencia lógica que busca una solución a un problema novedoso y Co que arroja datos sobre la capacidad inductiva, a partir de una característica implícita que otorga lógica a un conjunto determinado. Las tareas que requieren razonamiento fluido no verbal ponen en juego la capacidad de manejar conceptos abstractos, generalizaciones, relaciones lógicas, reglas y la capacidad para adaptarse con plasticidad a situaciones nuevas. Cuando se presentan pobres puntuaciones Gf-nv podría pensarse en dificultades para interpretar la información visual o efectuar predicciones basadas en la reflexión de dicha información (piezas y secuencias progresivas o dibujos ilustrativos). En otras palabras, las bajas puntuaciones obtenidas en este índice son un indicador de dificultades para descubrir características lógicas implícitas a un conjunto dado y/o inferir - a partir de reglas, premisas o condiciones - una secuencia lógica que redunde en una solución a un problema novedoso.

Para el índice Gf-v se consideran los subtests $\mathrm{S}$ y Ad, los cuales tienen como objetivo que el examinado identifique relaciones conceptuales abstractas entre dos o más palabras y exprese una respuesta verbal. La lectura de este índice permite reconocer los posibles vacíos en el conocimiento léxico que obstaculizan el razonamiento fluido, mientras que en otros casos puede ser que el examinado no logre organizar y analizar adecuadamente las semejanzas y las diferencias o en se encuentren dificultados los procesos de abstracciones. En los casos que el examinado no logre o muestre en diversos grados dificultades para elaborar conceptos abstractos es recomendable implementar un sondeo específico que torne al proceso fluido verbal más concreto y tangible; por ejemplo una lista de preguntas guiadas u objetos precisos como imágenes en lugar de utilizar estímulos abstractos (Brenlla, Taborda \& Labin, 2013).

La implementación de estas puntuaciones alternativas además de ahondar en los dominios fluidos, permite ampliar el análisis del perfil cuando el índice razonamiento perceptivo del test resulta pobre, errático o inconsistente. En estos casos, es recomendable que el examinador coteje estas puntuaciones considerando su consistencia con las de razonamiento fluido verbal y no verbal.

La difícil tarea de desarrollar diagnósticos diferenciales es prioritaria en el ámbito de la salud mental y su promoción. Tanto la sobrepatologización de la niñez y la adolescencia como la minimización de dificultades o déficit son modos contrapuestos con que pueden desembocar en prácticas iatrogénicas. La primera porque tienden a inmovilizar y la segunda porque al crear expectativas que no podrán alcanzarse tienden a culpabilizar.

Las dificultades socioeconómicas, exigencias en producción académica, carencias en la estimulación psicoeducativas, entre otras, en innumerables ocasiones se reflejan desfavorablemente en el desarrollo de procesos cognitivos que demanda de la puesta en juego de 
la inteligencia cristalizada. Desde este punto de vista contar con indicadores que permitan estudios minuciosos respecto de las habilidades fluidas es un aporte a la práctica de evaluación psicológica. Más aún si tenemos en cuenta que se trata de índices que pueden ser aplicables a un alto porcentaje de la población muestral.

Es importante destacar, que el análisis confirmatorio sobre la base de la teoría CHC realizado en el prueba original, arroja como uno de los cinco factores estructurales, el índice de razonamiento fluido (IRF) conformado por $\mathrm{M}$, Co y Ar que refiere a las operaciones mentales que utiliza un individuo cuando se enfrenta a una tarea relativamente nueva que no puede ser realizada de manera automática (Keith et al 2006). En nuestro país, se alcanzaron resultados disímiles ya que Ar mostró cargas compartidas con inteligencia cristalizada y memoria de corto plazo, por lo que estos factores parecen ser más importantes que los fluidos en la explicación del subtest (Brenlla, 2013). De este modo, en nuestra población el IRF que representa habilidades generales fluidas, coincide con el índice específico Gf-nv ya que se componen de las mismas tareas.

Por último, se encontró como limitación la falta de evidencia de validez de criterio externo. Sin embargo, como se indicó en los resultados, el análisis factorial confirmatorio permite sostener que la estructura de índices alternativos propuesta resulta significativa para interpretar las puntuaciones fluidas del test. Asimismo, se alcanzaron evidencias satisfactorias de consistencia interna, por lo cual se sostiene que se trata de índices estables y precisos como instrumentos de medición.

\section{Referencias}

Brenlla, M. E. (2013). Interpretación del WISCIV: Puntuaciones compuestas y modelos CHC. Ciencias Psicológicas, 7(2), 183-197. doi: http://dx.doi.org/10.22235/cp.v7i1

Brenlla, M. E., Taborda, A., \& Labin, A. (2013). Descripción del WISC-IV. En M. E. Brenlla y A. Taborda (Comps.) Guía para una interpretación integral del WISC-IV (pp.5658). Buenos Aires, Argentina: Paidós.

Carasatorrea, M., Ramírez-Amayaa, V., \& Díaz Cintra, S. (2016). Plasticidad sináptica estructural en el hipocampo inducida por la experiencia espacial y sus implicaciones en el procesamiento de información. Neurología, 31(8), 543-549. doi: 10.1016/j.nrl.2012.12.005

Carroll, J. B. (1993). Human cognitive abilities: A survey of factor analytic studies. New York: Cambridge University Press. Recuperado de http://steinhardtapps.es.its.nyu.edu/create/cour ses/2174/reading/Carroll_1.pdf

Cattell, R. B. (1963). Theory of fluid and crystallized intelligence: A critical experiment. Journal of Educational $\begin{array}{llll}\text { Psychology, } & 54 & \text { (1), } & \text { 1-22. }\end{array}$ doi.org/10.1037/h0046743

Coronel, C., Lacunza, B., \& Contini, N. (2006). Las habilidades cognitivas en niños privados culturalmente. Resultados preliminares de la primera fase de evaluación. Revista Iberoamericana de Diagnóstico y Evaluación - e Avaliação Psicológica, 22(2). Recuperado de http://www.aidep.org/03_ridep/R22/R223.pdf

Ferrer, E., O’Hare, E., \& Bunge, S. (2009) Fluid reasoning and the developing brain. Frontiers in neuroscience, $3(1), \quad 46$. doi.org/10.3389/neuro.01.003.2009

Flanagan, D. P., \& Kaufman, A. S. (2009). Claves para la evaluación con el WISC-IV. Mexico D.F., Mexico: Editorial el Manual Moderno (Ed. orig: Essentials of WISC-IV Assessment. John Wiley \& Sons. 2004)

Flanagan, D. P., Ortiz, S. O., Alfonso, V. C., \& Dynda, A. (2014). Cognitive assessment: Progress in psychometric theories, the structure of cognitive tests, and approaches to test interpretation. En D. Saklofske, V. Schwean, \& C. Reynolds (Eds.), Oxford handbook of psychological assessment of children and adolescents. New York: Oxford University Press.

Frank de Verthelyi, R. (1999). El análisis intra e intertest en la evaluación cognitiva: Aportes del Woodcock-Muñoz, R. a la batería cognitiva. Revista Iberoamericana de Diagnóstico y Evaluación Psicológica $\mathrm{E}$ Avaliação Psicológica, 1. Recuperado de http://www.aidep.org/03_ridep/R07/R071.pdf

George, D. \& Mallery, P. (2003). SPSS for Windows step by step: A simple guide and reference. 11.0 Update (4. ${ }^{\mathrm{a}}$ ed.). Boston: Allyn \& Bacon. 
Hair, J. F., Black, W. C., Babin, B. J., Anderson, R. E., \& Tatham, R. L. (2006). Multivariate data analysis (6th Edition). New Jersey: Pearson Education.

Horn, J. L., \& Noll, J. (1997). Human cognitive capabilities: Gf-Gc theory. En D. P. Flanagan, J. L. Genshaft \& P. L. Harrison (Eds.), Contemporary intellectual assessment: Theories, tests, and issues (pp. 53-91), Nueva York: Guilford Press.

Horn, J.L. (1991). Measurement of intellectual capabilities: A review of theory. En K.S. McGrew, J.K. Werder y R.W. Woodcock, Woodcock-Johnson Technical Manual (pp.197-232). Chicago: Riverside

Hu, L.T. \& Bentler, P.M. (1995). Fit indices in covariance structure modeling: Sensitivity to underparameterized model misspecification. Psychological Methods, 3, 424-453. doi.org/10.1037/1082-989X.3.4.424

Instituto Nacional de Estadísticas y Censos. (2001). Censo nacional de población, hogares $y$ vivienda 2001 [National census of population, household and housing]. Buenos Aires: Instituto Nacional de Estadísticas y Censos. Recuperado de http://www.indec.gob.ar/

Keith, T. Z. (1990). Confirmatory and hierarchical confirmatory analysis of the differential ability scales. Journal of Psychoeducational Assessment, 8(3), 391-405

Keith, T. Z., \& Reynolds, M. R. (2012). Using confirmatory factor analysis to aid in understanding the constructs measured by intelligence tests. In D. P. Flanagan \& P. L. Harrison (Eds.), Contemporary intellectual assessment: Theories, tests, and issues (3rd ed.). pp. 758-799. New York: Guilford.

Keith, T. Z., Fine, J. G., Taub, G., Reynolds, M. R., \& Kranzler, J. H. (2006). Higher Order, Multisample, Confirmatory Factor Analysis of the Weschler Intelligence Scale for ChildrenFourth Edition: What does It Measure? School Psychology Review, 35 (1), 108127. Recuperado de https://scholars.opb.msu.edu/en/publications/h igher-order-multisample-confirmatory-factoranalysis-of-the-wech-3

McGrew, K. S. (2009). CHC theory and the human cognitive abilities project: standing on the shoulders of the giants of psychometric intelligence research. Intelligence, 37(1), 110. doi.org/10.1016/j.intell.2008.08.004

Muñoz Céspedes J.; Tirapu Ustárroz J.; Ríos Lago M. \& Paúl Lapedriza, N. (Eds.) (2008). Neuroimagen. Técnicas y procesos cognitivos. doi: 10.1016/B978-84-458-1776-6.50023-X

Taborda, A. (2013). Enfoque relacional en el diagnóstico de niños derivados desde instituciones escolares. En A. Taborda, G. Dueñas, y G. Leoz (comps.) Paradojas que habitan las instituciones educativas en tiempos de fluidez. San Luis, Argentina: Nueva Editorial Universitaria UNSL. Recuperado de http://www.neu.unsl.edu.ar/?page_id=286

Taborda, A., Barbenza, C. \& Brenlla, M.E. (2011). Adaptación argentina del WISC-IV Wechsler. Escala de Inteligencia de Wechsler para niños cuarta edición (WISC-IV). Buenos Aires, Argentina: Paidós.

Tellegen, A. \& Briggs, P. (1967). Old wine in new skins: Grouping Wechsler subtests into new scales. Journal of Consulting Psychology, 31, 499-506. doi.org/10.1037/h0024963

Weiss, L. G., Saklofske, D. H., Holdnack, J. A., \& Prifitera, A. (2015). WISC-V: Advances in the assessment of intelligence. En L. Weiss, D. Saklofske, J. Holdnack \& A. Prifitera, WISC-V Assessment and Interpretation. (pp. 3-23) 


\section{Material Complementario. Índices alternativos de razonamiento fluido del WISC-IV}

1. Determinar si los índices de razonamiento fluido son unitarios.

1.1 Calcular la diferencia entre M y Co para determinar si el Gf-no verbal puede emplearse como medida resumen del razonamiento fluido sin enfatizar en las demandas verbales de las tareas

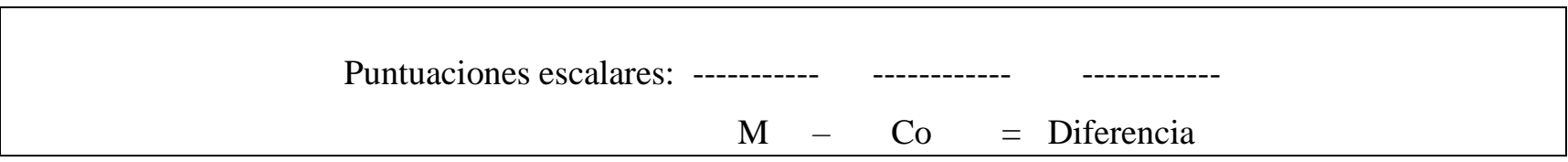

¿La diferencia es igual o mayor de 1.5 desvío estándar (5 puntos)?

\section{SI NO}

Si la respuesta es SI, el Gf-no verbal no debería interpretarse. Continuar con el paso 2.

Si la respuesta es NO, el Gf-no verbal puede interpretarse como una medida confiable y valida de la capacidad fluida del examinado.

1.1.1 Para calcular el Gf-no verbal, sumar las puntuaciones escalares de los dos subtests principales $M$ y

Co. Luego, recurrir a la Tabla 1 para determinar el Gf-no verbal

$$
\begin{aligned}
& \text {----- ------ = ------ = ---------------- } \\
& \mathrm{M}+\mathrm{Co}=\text { suma }=\text { Gf-no verbal }
\end{aligned}
$$

1.2 Calcular la diferencia entre $S$ y Ad para determinar si el Gf-verbal puede emplearse como una medida resumen del razonamiento fluido verbal

Puntuaciones escalares:

$\mathrm{S}-\mathrm{Ad}=$ Diferencia

¿La diferencia es igual o mayor de 1.5 desvío estándar (5 puntos)?

SI NO

Si la respuesta es SI, el Gf-verbal no debería interpretarse. Continuar con el paso 3.

Si la respuesta es NO, el Gf-verbal puede interpretarse como una medida confiable y valida de la competencia cognitiva del examinado.

1.3.1 Para calcular el Gf-verbal, sumar las puntuaciones escalares del subtest principal $S$ y el subtest optativo Ad. Luego, recurrir a la Tabla 2 para determinar el Gf-verbal

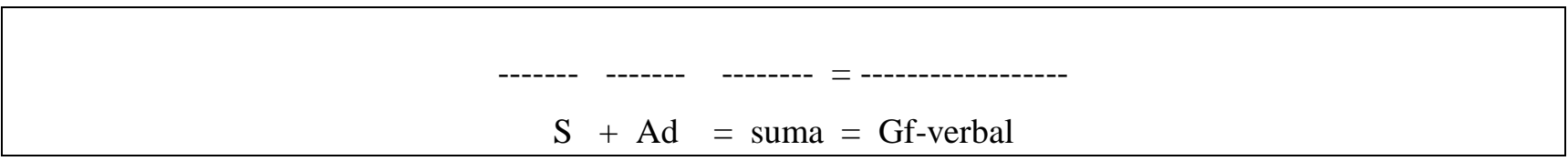

2. Calcular la magnitud de la diferencia entre Gf-no verbal y Gf-verbal (independientemente de la dirección)

$$
\text { Gf-no verbal }- \text { Gf-verbal }=\text { Diferencia }
$$

¿La diferencia es mayor de 23 puntos? SI NO

Si la respuesta es SI, la diferencia es infrecuente ya que se corresponde con el 10\% de la muestra

Si la respuesta es NO, la diferencia es frecuente

3. Describir los resultados de la comparación encontrada

Independientemente del resultado del paso anterior, es necesario generar un enunciado interpretativo que describa los resultados de la comparación entre los índices de razonamiento fluido. 